\title{
RECHAZO ESCOLAR Y OPTIMISMO Y PESIMISMO EN UNA MUESTRA DE ADOLESCENTES ECUATORIANOS
}

\author{
Aitana Fernández-Sogorb \\ Universidad de Alicante \\ aitana.fernandez@ua.es \\ Carlos Manuel Calderón Guevara \\ Universidad Central de Ecuador \\ chekalberto@yahoo.com \\ María del Pilar Aparicio-Flores \\ Universidad de Alicante \\ pilar.aparicio@ua.es \\ José Manuel García-Fernández \\ Universidad de Alicante \\ josemagf@ua.es
}

Fecha de Recepción: 8 Mayo 2019

Fecha de Admisión: 25 Septiembre 2019

\section{RESUMEN}

Tanto el rechazo escolar como el optimismo y el pesimismo son constructos ampliamente estudiados en el ámbito escolar por su impacto en el logro académico y el bienestar emocional de los estudiantes. Sin embargo, son escasos los estudios que han analizado la relación entre ambas variables. Este trabajo tuvo como objetivo analizar las diferencias en optimismo y pesimismo entre estudiantes con altas y bajas puntuaciones en rechazo escolar. 1,786 adolescentes ecuatorianos de entre 15 y 18 años $\left(M_{e d a d}=16.31 ; D E=1.01\right)$ respondieron a la School Refusal Assessment ScaleRevised for Children (SRAS-R-C) y al Youth Life Orientation Test (YLOT). La prueba $t$ de Student de diferencias de medias para muestras independientes reveló que los estudiantes con bajos niveles de rechazo escolar basado en la evitación de estímulos que producen afectividad negativa, en el escape de la aversión social y/o situaciones evaluativas, así como en la búsqueda de refuerzos tangibles fuera de la escuela, puntuaron significativamente más alto en optimismo. Por otra parte, los estudiantes con altos niveles de rechazo escolar por evitación de estímulos que producen afectividad negativa, por el escape de la aversión social y/o situaciones evaluativas y por la búsqueda de atención de personas significativas obtuvieron puntuaciones significativamente más altas en pesimismo. Estos resultados proporcionan información relevante para el diseño de programas de intervención en actitudes pesimistas del alumnado que manifiesta alto rechazo a la escuela.

Palabras clave: rechazo escolar; optimismo; pesimismo; bachillerato; adolescencia. 


\section{ABSTRACT}

School refusal and optimism and pessimism in an Ecuadorian adolescents' sample. Both school refusal and optimism and pessimism are constructs widely studied in the school setting because of their impact on student academic achievement and emotional well-being. However, there are few studies that have analyzed the relation between both variables. This work aimed to analyze the differences in optimism and pessimism among students with high and low scores on school refusal. 1,786 Ecuadorian adolescents aged 15 to $18\left(M_{a g e}=16.31 ; S D=1.01\right)$ answered to the School Refusal Assessment Scale-Revised for Children (SRAS-R-C) and the Youth Life Orientation Test (YLOT). Student's t-test of mean differences for independent samples revealed that students with low levels of school refusal based on the avoidance of stimuli that provoke negative affectivity, on the escape from social aversion and/or evaluative situations, as well as on the pursuit of tangible reinforcement outside of school, scored significantly higher on optimism. On the other hand, students with high levels of school refusal due to avoidance of stimuli that provoke negative affectivity, due to the escape from social aversion and/or evaluative situations and due to the pursuit of attention from significant others, obtained significantly higher scores on pessimism. These results provide relevant information for the design of intervention programs on pessimistic attitudes addressed to students who show high school refusal.

Keywords: school refusal; optimism; pessimism; baccalaureate; adolescence.

\section{INTRODUCCIÓN}

El término de rechazo escolar describe la negativa de un estudiante a asistir a la escuela y/o la dificultad para quedarse o permanecer durante las clases que dura la jornada escolar, pudiendo este comportamiento basarse 0 no en la ansiedad (Hendron y Kearney, 2011). Esta definición engloba términos tales como fobia escolar y absentismo (Kearney y Silverman, 1996).

Los jóvenes que muestran este tipo de comportamiento pueden experimentar alguna angustia emocional y, en particular, ansiedad; además se ha relacionado el rechazo escolar con varios trastornos de ansiedad como fobia específica, fobia social, trastorno de ansiedad generalizada y trastorno de ansiedad por separación (Beidel y Turner, 2005; Kearney y Albano, 2004 citado en Richards y Hadwin, 2011).

El rechazo escolar es un problema complejo y heterogéneo que consiste en numerosos síntomas de internalización y externalización, lo que determina graves consecuencias a corto y largo plazo como resultado del ausentismo escolar prolongado (Kearney, 2002). En este sentido, puede implicar ausencias completas o parciales de la escuela, tardanza crónica, mala conducta matutina en un intento de faltar a la escuela 0 una angustia sustancial en la escuela que precipita las súplicas para futuras ausencias (Kearney y Silverman 1996 citado en Haight, Kearney, Hendron y Schafer, 2011). Diversos estudios caracterizan a jóvenes con rechazo leve, grave, agudo y crónico, de acuerdo con el grado de frecuencia de las inasistencias o ausencias a la escuela (Kearney, Turner y Gauger, 2010; King, Ollendick y Tonge, 1995; Kearney, 2007; García Fernández et al., 2016).

Actualmente el modelo funcional se constituye en el cardinal enfoque para la clasificación del rechazo escolar (García Fernández et al., 2016). Este modelo fundamenta cuatro factores latentes: Eludir el afecto negativo que producen estímulos del contexto escolar, Escapar de la aversión social o situaciones de evaluación, Búsqueda de la atención de otras personas significativas y Búsqueda de refuerzos tangibles fuera de la escuela (Kearney y Silverman, 1996).

Por otra parte, la escuela debe trabajar en la formación emocional positiva de los adolescentes, con el propósito de fortalecer todas las potencialidades del desarrollo humano. En este marco, tanto el optimismo como el pesimismo han sido las variables más investigadas en el contexto escolar, ya que están relacionadas con el bienestar emocional de los estudiantes (Castillo, 2014). 
El optimismo y pesimismo constituyen características disposicionales de la personalidad, lo que implica que están implícitos en la manera en que cada persona manifiesta lo que acontece (Scheier y Carver, 1985; Seligman, 1991). En consecuencia, ser optimista implica una disposición en espera de que ocurran cuestiones positivas y, por el contrario, el pesimismo responde a una disposición en espera de que ocurran cuestiones negativas (Giménez, 2005).

El optimismo es un factor asociado a buen rendimiento académico, mejores relaciones interpersonales, bajos niveles de enfermedad y expectativas de alcanzar lo esperado (Fernández y Doldan, 2014). En cambio, el pesimismo es un factor asociado a actitudes problemáticas, bajo rendimiento, dificultades interpersonales, deterioro de la salud física (Seligman, Reivich, Jaycox y Gillham, 2005).

Revisada la literatura científica, son escasos los estudios que analizan el optimismo/pesimismo con el rechazo escolar basado en el modelo funcional. En las investigaciones de Gisbert-Ferrandiz, Vicent, Gisbert-Ferrandiz, Gonzálvez y Lagos-San Martín (2013) y Gonzálvez (2016) se evalúan estas variables. Los resultados obtenidos coinciden en que los factores Evitar el afecto negativo que produce estímulos o aspectos relacionados con el contexto escolar (Factor I), Escapar de la aversión social o situaciones de evaluación (Factor II) y Búsqueda de la atención de otras personas significativas (Factor III) de la School Refusal Assessment Scale Revised for Children (SRAS-R-C; Kearney, 2002), muestran correlaciones positivas y significativas en la dimensión pesimismo. En cambio, en el factor Búsqueda de refuerzos tangibles fuera de la escuela (Factor IV), existe correlación positiva y significativa en la dimensión optimismo.

\section{OBJETIVOS DE LA INVESTIGACIÓN}

El objetivo del presente estudio es analizar las diferencias en optimismo y pesimismo entre estudiantes con altas y bajas puntuaciones en rechazo escolar para cada uno de los factores y la puntuación total de la SRAS-R-C. Tomando como referencia la evidencia empírica previa existente (Gisbert-Ferrandiz et al., 2013; Gonzálvez, 2016), se espera que los estudiantes con alto rechazo escolar presenten puntuaciones significativamente inferiores en optimismo y significativamente superiores en pesimismo que sus iguales con bajo rechazo escolar.

\section{MÉTODO}

\section{Participantes}

Se llevó a cabo un muestreo aleatorio por conglomerados del que se obtuvo una muestra conformada por 1786 estudiantes de entre 15 y 18 años $\left(M_{e d a d}=16.31 ; D E=1.01\right)$. El $49 \%$ de los participantes eran chicas y el $51 \%$ chicos. Respecto a la distribución de la muestra en función del curso, se obtuvo que el $39.6 \%, 33.8 \%$ y el $26.6 \%$ de los participantes cursaban primero, segundo y tercero de bachillerato, respectivamente. En la Tabla 1 se puede observar la distribución de la muestra por sexo y curso académico. La prueba $\chi^{2}$ de la homogeneidad de la distribución de frecuencias reveló que no existían diferencias estadísticamente significativas para los seis grupos de sexo por edad $\left(\chi^{2}=.41 ; p=.81\right)$. 


\section{RECHAZO ESCOLAR Y OPTIMISMO Y PESIMISMO EN UNA MUESTRA DE ADOLESCENTES ECUATORIANOS}

Tabla 1

Número y porcentaje de sujetos de la muestra por sexo y curso académico.

\begin{tabular}{ccccc}
\hline \multicolumn{5}{c}{ Curso } \\
Sexo & $1^{\text {o }}$ & $2^{\text {o }}$ & $3^{\text {o }}$ & Total \\
\hline Chicos & 366 & 308 & 236 & 910 \\
$\%$ & $20.5 \%$ & $17.2 \%$ & $13.2 \%$ & $51.0 \%$ \\
Chicas & 342 & 296 & 238 & 876 \\
$\%$ & $19.1 \%$ & $16.6 \%$ & $13.3 \%$ & $49.0 \%$ \\
Total & 708 & 604 & 474 & 1786 \\
$\%$ & $39.6 \%$ & $33.8 \%$ & $26.6 \%$ & $100.0 \%$ \\
\hline
\end{tabular}

Nota: Estudiantes de primero, segundo y tercero de BGU.

\section{Medidas} 2002)

Rechazo escolar. School Refusal Assessment Scale Revised for Children (SRAS-R-C; Kearney,

La SRAS-R-C es una medida de autoinforme que consta de 24 ítems, los cuales evalúan varios tipos de rechazo escolar en niños y adolescentes. Esta escala tiene como objetivo medir la autopercepción del alumno en cuanto a cuatro factores justificativos del origen subyacente a la conducta de rechazo a la escuela. Estos factores son los siguientes: (I) Evitar la afectividad negativa que provocan los estímulos o situaciones relacionadas con el ámbito escolar, (II) Escapar de la aversión social o situaciones de evaluación; (III) Búsqueda de la atención de otras personas significativas, y (IV) Búsqueda de refuerzos tangibles fuera del ámbito escolar. La escala de respuesta es de tipo Likert y contiene 7 puntos ( $0=$ Nunca; $6=$ Siempre). Los niveles de fiabilidad que se obtuvieron en la versión española de la SRAS-R-C oscilaron entre .70 y .87 para sus factores (Gonzálvez et al., 2016).

Optimismo/pesimismo. Test de Orientación en la vida para jóvenes (Youth Life Orientation Test, YLOT; Ey et al., 2005)

EI YLOT tiene como finalidad evaluar las expectativas positivas (Optimismo) y negativas (Pesimismo). Desde la dimensión Optimismo, los objetivos propuestos son percibidos como metas alcanzables, mientras que el Pesimismo percibe los objetivos como hechos imposibles o con dificultad para ser. La fiabilidad reportada en el estudio desarrollado por Ey et al. (2005) consiste en valores de consistencia interna de .79 para Optimismo, .78 para Pesimismo.

\section{Procedimiento}

Primeramente se entrevistó a los directores de los centros educativos que participaron en el estudio, con el fin de exponer los objetivos de la investigación, describir las medidas de autoinforme a utilizar y solicitar su consentimiento informado. Posteriormente, se llevó a cabo una reunión con los padres de los adolescentes para explicarles igualmente los objetivos del estudio y solicitarles su consentimiento por escrito por el que autorizaban a sus hijos a participar. A continuación se procedió a administrar los instrumentos de evaluación a los participantes dentro del aula ordinaria y asegurándoles en todo momento la voluntariedad y el anonimato de las pruebas. Uno de los investigadores estuvo presente en el momento de la aplicación de las medidas de autoinforme para resolver posibles dudas.

\section{Análisis de datos}

Se empleó la prueba $t$ de Student de diferencias de medias para muestras independientes. Para establecer la magnitud de las diferencias halladas, se recurrió al índice $d$ (diferencia de media tipificada). La interpretación del mismo se realizó atendiendo a los criterios establecidos por Cohen 
(1988): tamaño pequeño (valores entre .20 y .50), moderado (valores entre .51 y .79) y grande (valores $\geq .80$ ). Los análisis fueron realizados con el programa informático SPSS/IBM versión 22.0.

\section{RESULTADOS ALCANZADOS}

La Tabla 2 y Figura 1 muestran las medias y diferencias estadísticamente significativas obtenidas para las variable Optimismo y Pesimismo en estudiantes con altas y bajas puntuaciones en rechazo escolar para el Factor I de la SRAS-R-C, negativa a asistir a la escuela debido a Evitar la afectividad negativa que provocan los estímulos o situaciones relacionadas con el ámbito escolar. Las diferencias halladas entre los dos grupos resultaron significativas para ambas dimensiones. Por un lado, los estudiantes con bajos niveles de rechazo escolar obtuvieron puntuaciones más altas que sus iguales con altos índices de rechazo en Optimismo. Mientras que los estudiantes con altas puntuaciones en rechazo escolar obtuvieron una puntuación mayor que sus iguales con bajos niveles de rechazo en Pesimismo. El tamaño de las diferencias halladas fue pequeño tanto para la dimensión de Optimismo $(d=.23)$ y para el Pesimismo $(d=.48)$.

Tabla 2

Diferencias en optimismo y pesimismo en estudiantes con altas y bajas puntuaciones en el Factor I de la SRAS-R-C

\begin{tabular}{|c|c|c|c|c|c|c|c|c|c|c|}
\hline \multirow[t]{2}{*}{ Variable } & \multicolumn{2}{|c|}{$\begin{array}{l}\text { Prueba } \\
\text { Levene }\end{array}$} & \multicolumn{2}{|c|}{$\begin{array}{c}\text { Altas } \\
\text { puntuaciones }\end{array}$} & \multicolumn{2}{|c|}{$\begin{array}{c}\text { Bajas } \\
\text { puntuaciones }\end{array}$} & \multicolumn{4}{|c|}{$\begin{array}{l}\text { Significación estadística y } \\
\text { magnitud diferencias }\end{array}$} \\
\hline & $F$ & $p$ & $M$ & $D E$ & $M$ & $D E$ & $t$ & g.l. & $p$ & $d$ \\
\hline $\mathrm{O}$ & .01 & .908 & 12.32 & 3.31 & 13.07 & 3.32 & 3.66 & 1048 & $<.001$ & .23 \\
\hline $\mathrm{P}$ & .01 & .916 & 8.57 & 4.06 & 6.62 & 3.98 & 7.85 & 1048 & $<.001$ & .48 \\
\hline
\end{tabular}

Nota: Factor I SRAS = Evitar la afectividad negativa que provocan estímulos o situaciones relacionadas con el ámbito escolar; $\mathrm{O}=$ Optimismo; $\mathrm{P}=$ Pesimismo.

Figura 1. Diagrama de barras para las diferencias en puntuaciones de optimismo y pesimismo en estudiantes con altas y bajas puntuaciones en el Factor I de la SRAS-R-C. ${ }^{*}=p<.05 ;{ }^{* *}=p<.01 ;{ }^{* * *}=p<.001$

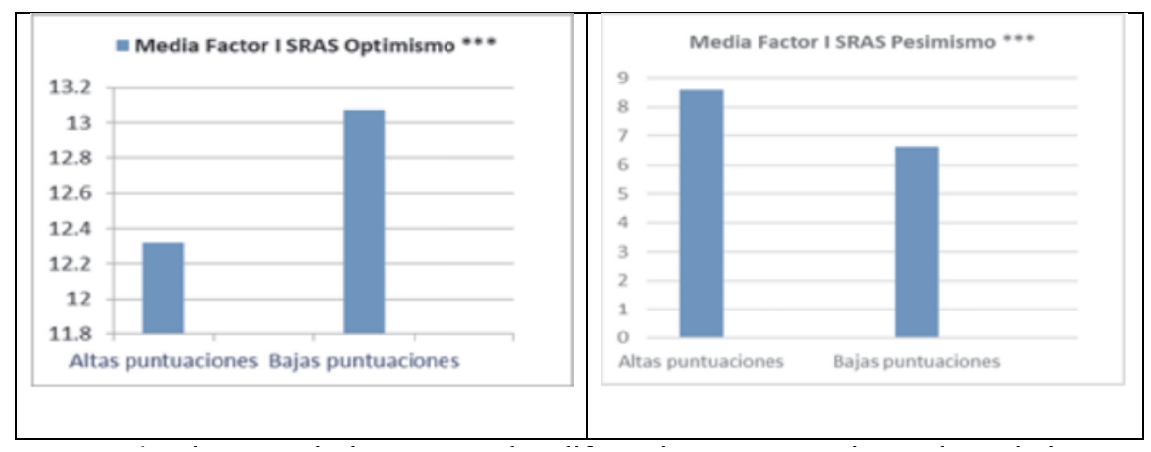

La Tabla 3 y Figura 2 muestran las medias y diferencias estadísticamente significativas obtenidas para las variable Optimismo y Pesimismo en estudiantes con altas y bajas puntuaciones en rechazo escolar para el Factor II de la SRAS-R-C, negativa a asistir a la escuela debido a Escapar de la aversión social o situaciones de evaluación. Las diferencias halladas entre los dos grupos resultaron significativas para ambas dimensiones. Por un lado, los estudiantes con bajos niveles de rechazo escolar obtuvieron puntuaciones más altas que sus iguales con altos índices de rechazo en 
Optimismo. Mientras que los estudiantes con altas puntuaciones en rechazo escolar obtuvieron una puntuación mayor que sus iguales con bajos niveles de rechazo en Pesimismo. El tamaño de las diferencias halladas fue pequeño para la dimensión de Optimismo $(d=.41)$ y moderado para el Pesimismo $(d=.67)$.

Tabla 3

Diferencias en optimismo y pesimismo en estudiantes con altas y bajas puntuaciones en el Factor II de la SRAS-R-C

\begin{tabular}{ccccccccccc}
\hline & \multicolumn{2}{c}{$\begin{array}{c}\text { Prueba } \\
\text { Variable }\end{array}$} & \multicolumn{2}{c}{$\begin{array}{c}\text { Altas } \\
\text { Levene }\end{array}$} & \multicolumn{2}{c}{$\begin{array}{c}\text { Bajas } \\
\text { puntuaciones }\end{array}$} & \multicolumn{4}{c}{$\begin{array}{c}\text { Significación estadística y } \\
\text { puntuaciones }\end{array}$} \\
\hline & $F$ & $p$ & $M$ & $D E$ & $M$ & $D E$ & $t$ & g.l. & $p$ & $d$ \\
\hline $\mathrm{O}$ & .80 & .369 & 11.98 & 3.35 & 13.34 & 3.22 & 6.63 & 1042 & $<.001$ & .41 \\
$\mathrm{P}$ & .55 & .456 & 8.92 & 3.86 & 6.30 & 3.94 & 10.82 & 1042 & $<.001$ & .67 \\
\hline
\end{tabular}

Nota: Factor II SRAS = Escapar de la aversión social o situaciones de evaluación; $\mathrm{O}=$ Optimismo; $\mathrm{P}=$ Pesimismo.

Figura 2. Diagrama de barras para las diferencias en puntuaciones de optimismo y pesimismo en estudiantes con altas y bajas puntuaciones en el Factor II de la SRAS-R-C. ${ }^{*}=p<.05 ;{ }^{* *}=p<.01 ;{ }^{* * *}=p<.001$

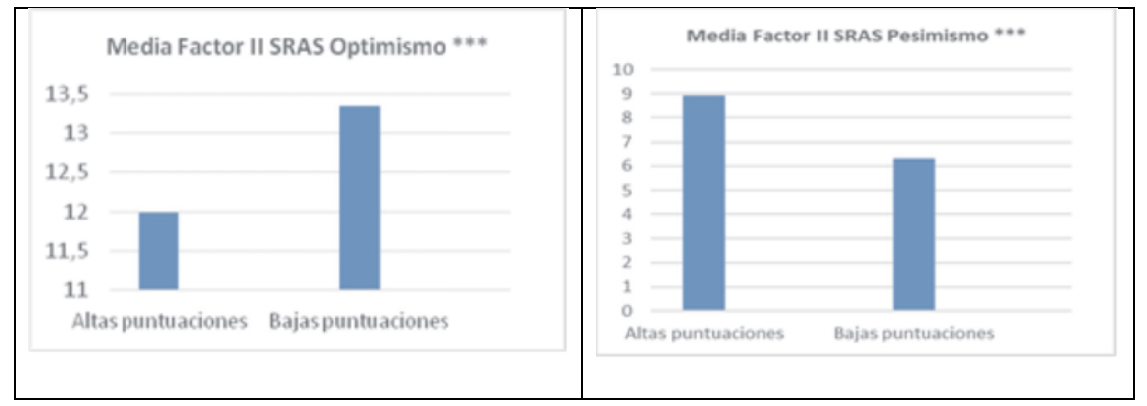

La Tabla 4 y Figura 3 muestran las medias y diferencias estadísticamente significativas obtenidas para las variable Optimismo y Pesimismo en estudiantes con altas y bajas puntuaciones en rechazo escolar para el Factor III de la SRAS-R-C, negativa a asistir a la escuela debido a Búsqueda de la atención de otras personas significativas. Los estudiantes con altas puntuaciones en rechazo escolar obtuvieron una puntuación significativamente mayor que sus iguales con bajos niveles de rechazo en Pesimismo con una magnitud de la diferencia pequeño $(d=.27)$, mientras que para la dimensión de Optimismo no se hallaron diferencias estadísticamente significativas.

Tabla 4

Diferencias en optimismo y pesimismo en estudiantes con altas y bajas puntuaciones en el Factor III de la SRAS-R-C

\begin{tabular}{crrrrrrrrrr}
\hline & \multicolumn{2}{c}{$\begin{array}{c}\text { Prueba } \\
\text { Variable }\end{array}$} & \multicolumn{2}{c}{$\begin{array}{c}\text { Altas } \\
\text { Levene }\end{array}$} & \multicolumn{2}{c}{$\begin{array}{c}\text { Bajas } \\
\text { puntuaciones }\end{array}$} & \multicolumn{3}{c}{$\begin{array}{c}\text { Significación estadística y } \\
\text { puntuaciones }\end{array}$} & \multicolumn{4}{c}{ magnitud diferencias } \\
\hline & $F$ & $p$ & $M$ & $D E$ & $M$ & $D E$ & $t$ & $g . l$. & $p$ & $d$ \\
\hline $\mathrm{O}$ & 3.66 & .056 & 12.67 & 3.25 & 12.70 & 3.49 & .13 & 990 & .897 & - \\
$\mathrm{P}$ & .01 & .956 & 8.06 & 4.11 & 6.98 & 4.01 & 4.09 & 990 & $<.001$ & .27 \\
\hline
\end{tabular}

Nota: Factor III SRAS = Búsqueda de la atención de otras personas significativas; $\mathrm{O}=$

Optimismo; $\mathrm{P}=$ Pesimismo. 
Figura 3. Diagrama de barras para las diferencias en puntuaciones de optimismo y pesimismo en estudiantes con altas y bajas puntuaciones en el Factor III de la SRAS-R-C. ${ }^{*}=p<.05 ;{ }^{* *}=p<.01 ;{ }^{* * *}=p<.001$

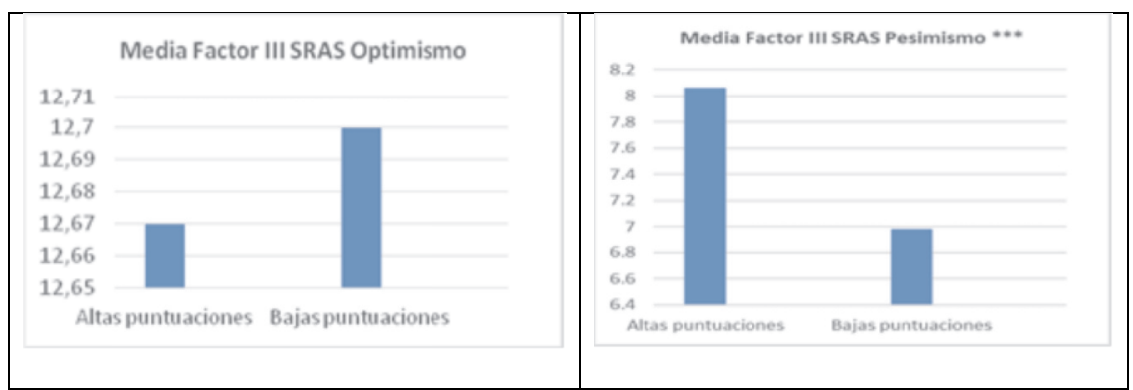

La Tabla 5 y Figura 4 muestran las medias y diferencias estadísticamente significativas obtenidas para las variable Optimismo y Pesimismo en estudiantes con altas y bajas puntuaciones en rechazo escolar para el Factor IV de la SRAS-R-C, negativa a asistir a la escuela debido a Búsqueda de refuerzos tangibles fuera de la escuela. Los estudiantes con bajos niveles de rechazo escolar obtuvieron puntuaciones significativamente más altas que sus iguales con altos índices de rechazo en Optimismo y la magnitud de la diferencia fue baja $(d=.43)$, mientras que para la variable Pesimismo no se hallaron diferencias estadísticamente significativas.

\section{Tabla 5}

Diferencias en optimismo y pesimismo en estudiantes con altas y bajas puntuaciones en el Factor IV de la SRAS-R-C

\begin{tabular}{ccccccccccc}
\hline Variable & \multicolumn{2}{c}{$\begin{array}{c}\text { Prueba } \\
\text { Levene }\end{array}$} & \multicolumn{2}{c}{$\begin{array}{c}\text { Altas } \\
\text { puntuaciones }\end{array}$} & \multicolumn{2}{c}{$\begin{array}{c}\text { Bajas } \\
\text { puntuaciones }\end{array}$} & \multicolumn{4}{c}{$\begin{array}{c}\text { Significación estadística y } \\
\text { magnitud diferencias }\end{array}$} \\
\hline & $F$ & $p$ & $M$ & $D E$ & $M$ & $D E$ & $t$ & $g . l$. & $p$ & $d$ \\
\hline $\mathrm{O}$ & 21.65 & .000 & 13.41 & 2.93 & 12.02 & 3.77 & 6.35 & 960 & $<.001$ & .43 \\
$\mathrm{P}$ & .04 & .831 & 7.23 & 4.11 & 7.56 & 4.09 & 5.89 & 560.17 & $<.001$ & - \\
\hline
\end{tabular}

Nota: Factor IV SRAS $=$ Búsqueda de refuerzos tangibles fuera de la escuela; $\mathrm{O}=$ Optimismo; $\mathrm{P}=$ Pesimismo.

Figura 4. Diagrama de barras para las diferencias en puntuaciones de optimismo y pesimismo en estudiantes con altas y bajas puntuaciones en el Factor IV de la SRAS-R-C. ${ }^{*}=p<.05 ;{ }^{* *}=p<.01 ;{ }^{* \star *}=p<.001$

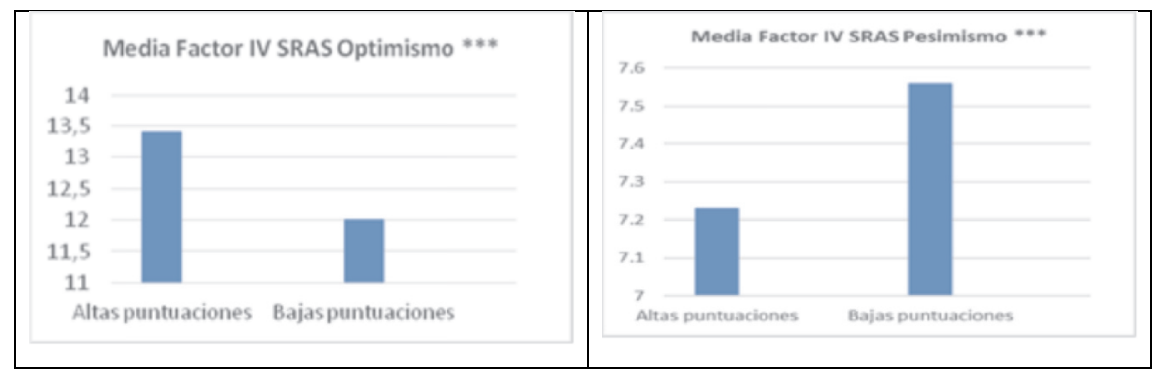




\section{DISCUSIÓN}

Este trabajo se propuso analizar las diferencias en optimismo y pesimismo entre estudiantes con altas y bajas puntuaciones en rechazo escolar. En este sentido, las diferencias de medias obtenidas en optimismo y pesimismo entre los estudiantes con altas y bajas puntuaciones en rechazo escolar correspondiente al Factor I Evitar la afectividad negativa que provocan estímulos o situaciones relacionadas con el ámbito escolar y al Factor II Escapar de la aversión social o situaciones de evaluación confirman la hipótesis, según la cual se esperaba que los estudiantes con alto rechazo escolar presentarían puntuaciones significativamente inferiores en optimismo y significativamente superiores en pesimismo que sus iguales con bajo rechazo escolar. Esto se debe a que los estudiantes con bajos niveles de rechazo escolar obtuvieron puntuaciones más altas en optimismo que sus iguales con altos índices de rechazo y significativamente superiores en pesimismo. Para el Factor III Búsqueda de la atención de otras personas significativas, la hipótesis se rechaza. Aunque en pesimismo sí se producen las diferencias en el sentido esperado, es decir los sujetos con alto rechazo escolar puntúan significativamente más alto que sus compañeros con bajas puntuaciones, en optimismo no existen diferencias estadísticamente significativas entre los dos grupos. Con relación al Factor IV Búsqueda de refuerzos tangibles fuera del ámbito escolar, también se rechaza la hipótesis, puesto que no se han hallado diferencias estadísticamente significativas en pesimismo. En optimismo se han identificado diferencias en sentido contrario, es decir, los sujetos con alto rechazo escolar puntuaron significativamente más alto.

Los hallazgos obtenidos para el Factor I Evitar la afectividad negativa que provocan estímulos o situaciones relacionadas con el ámbito escolar, Factor II Escapar de la aversión social o situaciones de evaluación y Factor III Búsqueda de la atención de otras personas significativas, concuerdan con los resultados de varios estudios que confirman las correlaciones positivas y significativas en la dimensión pesimismo. En cambio en el Factor IV Búsqueda de refuerzos tangibles fuera de la escuela, existe correlación positiva y significativa en la dimensión optimismo (Gisbert-Ferrandis et al., 2013; Gonzálvez, 2016).

Es importante plantear algunas limitaciones identificadas en este estudio que precisan ser analizadas en futuras líneas de investigación. En primer lugar, el diseño transversal de este estudio no permite hacer un análisis del comportamiento de rechazo escolar en los grupos investigados en una línea de tiempo diferente. Esta situación motiva a realizar estudios longitudinales, dada su escasez, sobre esta problemática, lo que permitiría obtener datos más precisos y concluyentes en las relaciones entre estos constructos, así como hacer inferencias respecto al cambio, determinantes y consecuencias del comportamiento de la negativa de asistir a la escuela por parte de niños y adolescentes. Existen estudios longitudinales que evalúan el absentismo escolar (Attwood y Croll, 2005; Dembo et al., 2013 citado en Gonzálvez, 2016), pero no se relaciona con la conducta de rechazo escolar.

En segundo lugar, el tamaño de la muestra constituye una limitación para la investigación, puesto que solo se incluyeron tres centros educativos, lo que dificulta hacer generalizaciones de los resultados a toda la población escolar ecuatoriana. Por ello, los futuros trabajos que evalúen esta problemática, deberían incluir instituciones educativas no solo de sostenimiento fiscal sino también municipal, fiscomisional y particular de diversas áreas geográficas del país.

En tercer lugar, los escasos estudios previos hallados sobre la relación entre el rechazo escolar y el optimismo y pesimismo, ha dificultado contraponer los hallazgos obtenidos. En este sentido, se propone consolidar el estudio en las variables investigadas y ampliar el análisis a otras variables que evidencian relación con el comportamiento de rechazo escolar, como son variables personales y del contexto, tales como motivación, autoestima, asertividad, empatía, problemas de conducta 
externalizantes, relaciones familiares, ambiente escolar, acoso escolar, dificultades de aprendizaje y rendimiento académico.

En general, los resultados obtenidos en esta investigación evidencian la relación existente entre el pesimismo y los altos niveles de rechazo escolar. Algunos estudios que correlacionan estas variables con la ansiedad (Higa, Daleiden y Chorpita, 2002; Kearney y Silverman, 1993 citado en Gonzálvez, 2016), plantean la necesidad de establecer estrategias de intervención orientadas al control y disminución de conductas emocionales negativas, tales como fobia escolar, fobia social, angustia, rabietas, agresividad, tristeza y culpabilidad.

Finalmente, se confía que este estudio motive a los diferentes profesionales ligados al campo de la psicología y la educación, autoridades del Estado responsables en diseñar las políticas educativas y padres de familia, a que razonen sobre esta problemática que afecta al desarrollo emocional, escolar, cultural y social de los niños y adolescentes que presentan estás dificultades; reflexionen sobre las causas y/o factores que originan este comportamiento, íntimamente ligado, como se mencionó anteriormente, a los altos índices de fracaso y decersión escolar, que inciden indudablemente en el desarrollo del Ecuador. Bajo este análisis, se presenta uno de los grandes desafíos que tiene el sistema educativo ecuatoriano.

\section{CONCLUSIONES}

Los estudiantes con altas puntuaciones en rechazo escolar para los Factores I Evitar la afectividad negativa que provocan estímulos o situaciones relacionadas con el ámbito escolar y II Escapar de la aversión social o situaciones de evaluación, presentaron menores puntuaciones en optimismo y mayores puntuaciones en pesimismo. Los estudiantes con altas puntuaciones en rechazo escolar para el Factor III Búsqueda de la atención de otras personas significativas, presentaron mayores puntuaciones en pesimismo; en cambio, no se encontraron diferencias significativas en rechazo escolar en optimismo. Por último, no se hallaron diferencias significativas en rechazo escolar para el Factor IV, Búsqueda de refuerzos tangibles fuera de la escuela en pesimismo, pero los estudiantes con altas puntuaciones en rechazo escolar presentaron mayores puntuaciones en optimismo en el mismo factor.

\section{REFERENCIAS BIBLIOGRÁFICAS}

Attwood, G. y Croll, P. (2015). Truancy and well-being among secondary school pupils in England. Educational Studies, 41(1), 14-28. doi: 10.1080/03055698.2014.955725

Beidel, D. C. y Turner, S. M. (2005). Childhood anxiety disorders: A guide to research and treatment. Nueva York: Routledge.

Castillo, D. (2014). Proyecto de intervención para la promoción del optimismo en niños de dos y tres años. Universidad Internacional de la Rioja. Facultad de Educación. Trabajo de Fin de Grado.

Cohen, J. (1988). Statistical power analysis for the behavioral sciences. Hillsdale, NJ: Erlbaum.

Dembo, R., Briones-Robins, R., Barrett, K., Winters, K. C., Schmeidler, J., Ungaro, R. A., Karas, L., Belenko, S. y Gulledge, L. (2013). Mental health, substance use, and delinquency among truant youth in a brief intervention Project: a longitudinal study. Journal of Emotional and Behavioral Disorders, 21(3), 176-192. doi: 10.1177/1063426611421006

Ey, S., Hadley, W., Allen, D. N., Palmer, S., Klosky, J., Deptula, D., Thomas, J. y Cohen, R. (2005). Una nueva medida del optimismo y el pesimismo de los niños: la orientación de la vida juvenil. Journal of Child Psychology and Psychiatry, 46(5), 548-58.

Fernández, C. y Doldan, N. (2014). El papel de las emociones positivas: Optimismo y Pesimismo en alumnos de educación social. Revista Electrónica de Investigación y Docencia (REID), 8, 35-54.

García-Fernández, J. M., Inglés, C. J., Gonzálvez, C., Vicent, M., Delgado, B. y Gómez-Núñez, M. I. (2016). Revisión bibliométrica del rechazo escolar: perspectivas de investigación y su análisis. Education Siglo XXI, 1, 71-92. 
Giménez, M. (2005). Optimismo y Pesimismo: variables asociadas en el contexto escolar. Pulso, 28, 9-23.

Gisbert-Ferrándiz, B., Vicent, J., Gisbert-Ferrándiz, A., Gonzálvez, C. y Lagos-San Martín, N. (2013) Optimismo, pesimismo y rechazo escolar. En J.M. García-Fernández (Coord.), Rechazo escolar y variables cognitivo-motivacionales y de personalidad. Simposio llevado a cabo en el Symposium Nacional de Psicología Clínica y de la Salud con Niños y Adolescentes, Elche, España.

Gonzálvez, C. (2016). Rechazo escolar en educación primaria y su relación con variables psicoeducativas. Tesis presentada para aspirar al grado de Doctora por la Universidad de Alicante mención de Doctora Internacional.

Gonzálvez, C., Inglés, C. J., Kearney, C. A., Vicent, M., San Martín, R. y García-Fernández, J. M. (2016). Escala de evaluación de rechazo escolar revisada: Invariancia factorial y diferencias de medios latentes a través del género y la edad en niños españoles. Frontiers in Psychology, 7, 1-11.

Haight, C., Kearney, C. A., Hendron, M. y Schafer, R. (2011). Análisis confirmatorios de la Escala de Evaluación de Rechazo Escolar Revisada: Replicación y Extensión a una Muestra de Absentismo Escolar. Journal of Psychopathology and Behavioral Assessment, 33, 196-204. doi:10.1007/s10862-011-9218-9.

Hendron, M. y Kearney, C. A. (2011). Bridging the gap between assessment and treatment of youths with school refusal behavior: what to do when clients ask "what now"? Journal of Clinical Psychology Practice, 2, 14-21.

Higa, C. K., Daleiden, E. L. y Chorpita, B. F. (2002). Psychometric properties and clinical utility of the School Refusal Assessment Scale in a multiethnic sample. Journal of Psychopathology and Behavioral Assessment, 24(4), 247-258. doi: 10.1023/A:1020727016113

Kearney, C. A. (2002). Identifying the function of school refusal behavior: A revision of the School Refusal Assessment Scale. Journal of Psychopathology and Behavioral Assessment, 24(4), 235245. doi: 10.1023/A:1020774932043

Kearney, C. A. (2007). Getting your child to say yes to school. Nueva York: Oxford University Press.

Kearney, C. A. y Albano, A. (2004). The functional profiles of school refusal behavior: Diagnostic aspects. Behavior Modification, 28(1), 147-161. doi: 10.1177/01454455033259263

Kearney, C. A. y Silverman, W. K. (1990). A preliminary analysis of a functional model of assessment and treatment for school refusal behavior. Behavior Modification, 14, 340-366.

Kearney, C. A. y Silverman, W. K. (1996). The evolution and reconciliation of taxonomic strategies for school refusal behavior. Clinical Psychology-Science and Practice, 3(4), 339-354. doi: 10.1111/j.1468-2850. 1996.tb00087.x

Kearney, C. A., Turner, D. y Gauger, M. (2010). School refusal behavior. En I. Weiner y E. Craighead

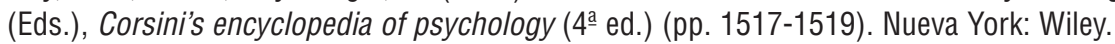

King, N. J., Ollendick, T. H. y Tonge, B. J. (1995). School refusal: Assessment and treatment. Boston, MA: Allyn \& Bacon.

Richards, H. J. y Hadwin, J. A. (2011). An exploration of the relationship between trait anxiety and school attendance in young people. School Mental Health, 3(4), 236-244. doi: 10.1007/s12310011-9054-9

Scheier, M. F. y Carver, C. S. (1985). Optimism, coping, and health: Assessment and implications of generalized outcome expectancies. Health Psychology, 4(3), 219-247. doi: 10.1037/02786133.4.3.219

Seligman, M. E. (1991). Learned optimism. New York: Knopf.

Seligman, M. E., Reivich, K., Jaycox, L. y Gillham, J. (2005). Niños optimistas. Barcelona: Grijalbo. 